\title{
Long-term Safety and Effectiveness of Tacrolimus in Patients With Lupus Nephritis: 5-year Interim Postmarketing Surveillance Study in Japan (TRUST)
}

\author{
Tsutomu Takeuchi ${ }^{1}(\mathbb{D})$, Naoko Wakasugi ${ }^{2}$ (D) Satoshi $\mathrm{Uno}^{2}$ (D) and Hirofumi Makino ${ }^{3}$ (D)
}

\begin{abstract}
Objective. To assess the long-term safety and effectiveness of tacrolimus for treating lupus nephritis (LN) in the real-world clinical setting.

Methods. This is an ongoing, open-label, noncomparative, observational, postmarketing surveillance study conducted across 275 sites in Japan. Registered patients with LN were followed for 10 years. Here we report data relating to 5 years of tacrolimus maintenance therapy at the interim data cutoff in August 2016.

Results. Of 1395 registered patients, 1355 received tacrolimus maintenance therapy for LN and provided safety data. The most common serious adverse drug reactions (ADR) included pneumonia (1.1\%), herpes zoster $(1.0 \%)$, cellulitis (1.0\%), and diabetes mellitus (1.0\%). ADR occurred mainly within the first 28 weeks of tacrolimus treatment, and no marked increase was observed during the follow-up period. Subgroup analyses suggested that risk factors for commonly observed ADR associated with tacrolimus included inpatient management, LN disease severity, increasing age, abnormal renal or hepatic function, and comorbid or previous disease. The cumulative rate of progression to renal failure (based on the attending physician's assessment) was $0.8 \%$ at Year 1 and $6.6 \%$ at Year 5. Cumulative relapse rates were $7.8 \%$ and 30.6\%, respectively. Urine protein:creatinine ratio, serum anti-dsDNA antibody levels, complement C3 levels, and steroid-sparing effects were all significantly improved from 4 weeks after tacrolimus treatment initiation $(P<0.001)$ and were sustained over 5 years.

Conclusion. Long-term tacrolimus maintenance treatment over 5 years in the real-world clinical setting was well tolerated and effective in a large population of patients with LN (www.ClinicalTrials.gov: NCT01410747).
\end{abstract}

Key Indexing Terms: immunosuppressive agent, lupus nephritis, postmarketing product surveillance

Systemic lupus erythematosus (SLE) is a multisystem autoimmune disorder with wide-reaching effects on most organ systems, including the kidneys. Approximately $40-70 \%$ of patients with SLE will develop lupus nephritis $(\mathrm{LN})^{1}$, which is associated with substantial patient morbidity and mortality, particularly in certain populations, such as black and Asian ethnic group ${ }^{2,3,4,5}$.

The pathogenesis of LN is highly complex and believed to be closely related to both systemic and intrarenal events ${ }^{6,7,8,9}$. Genetic predisposition, proinflammatory and antiinflammatory

This work was supported by Astellas Pharma, Inc. Editorial support was funded by Astellas Pharma, Inc.

${ }^{I}$ T. Takeuchi MD, PhD, Division of Rheumatology, Department of Internal Medicine, Keio University School of Medicine, Tokyo; ${ }^{2}$ N. Wakasugi, MSc, S. Uno, MSc, Astellas Pharma Inc., Tokyo; ${ }^{3}$ H. Makino MD, PhD, Okayama University, Okayama-Shi, Japan.

All authors report nonfinancial support from Astellas during the development of the manuscript. N. Wakasugi and S. Uno are employees of Astellas.

Full Release Article. For details see Reprints and Permissions at jrheum.org.

Address correspondence to N. Wakasugi, Astellas Pharma Inc.,

2-5-1, Nihonbashi-Honcho, Chuo-Ku, Tokyo 103-8411, Japan.

Email:naoko.wakasugi@astellas.com.

Accepted for publication March 10, 2020. cytokines, and defects in the complement system all have putative roles in the development of LN. In addition to these pathogenic factors, the role of autoantibodies, such as the anti-dsDNA antibody and aberrations in lymphocyte subsets, cannot be overemphasized in the pathogenesis of LN, and are amenable to immunosuppressive treatments 5 . Aberration of $\mathrm{T}$ lymphocytes, especially the T-helper (Th) subsets including Th1, Th2, Th9, Th 17, regulatory $T$ cells, and follicular Th cells, as well as B lymphocyte aberrations, are reported to play a crucial role in the pathogenesis of $\mathrm{LN}^{5}$.

The treatment of LN mainly involves remission induction therapy in the acute stage followed by maintenance therapy ${ }^{10}$. Cytotoxic agents, such as cyclophosphamide (CYC), in combination with corticosteroids, are the standard of care for LN treatment but are associated with considerable morbidity and suboptimal outcomes ${ }^{11}$. Immunosuppressants, such as azathioprine (AZA), mycophenolate mofetil (MMF), and cyclosporine (CSA), can also be used to achieve better control of disease activity, with corticosteroids used as the basis of all regimens ${ }^{12}$. However, these therapies are associated with efficacy and safety concerns, and a lack of supporting evidence ${ }^{12}$.

Tacrolimus, an immunosuppressive macrolide that blocks $\mathrm{T}$ cell activation by specifically inhibiting calcineurin, is widely 
administered following organ transplantation ${ }^{13}$. Tacrolimus therapy is considered to be a promising treatment option for LN due to associated improvements in the aberrational activation of T lymphocytes, especially Th subsets in LN. Following several randomized studies evaluating the efficacy and safety of tacrolimus as maintenance treatment for $\mathrm{LN}^{14,15}$, tacrolimus was approved for LN treatment in Japan in 2007, and subsequently in other Asian countries. However, data concerning the longterm safety and effectiveness of tacrolimus in patients with LN in a real-world clinical setting are lacking. This postmarketing surveillance (PMS) study was undertaken in Japan to assess the long-term safety and effectiveness of tacrolimus for maintenance treatment of LN in the real-world clinical setting, with patients being followed for up to 10 years. Here, we report the interim results of a 5-year analysis, involving 1355 registered patients.

\section{MATERIALS AND METHODS}

Study design. A Study to Evaluate the Safety and Efficacy of Tacrolimus for Lupus Nephritis Under Actual Use Situations (TRUST; ClinicalTrials.gov: NCT01410747) was an open-label, noncomparative, noninterventional, observational PMS study. TRUST was designed to prospectively assess the long-term safety and effectiveness of tacrolimus as maintenance therapy for LN in the real-world clinical setting, and aimed to include all sites in Japan with the potential to prescribe tacrolimus for LN. Ultimately, patients with LN initiating maintenance treatment with tacrolimus at 275 hospitals and clinics across Japan were registered centrally in an all-patient investigation system between January 2007 and January 2010. Registered patients were followed for 10 years, with planned safety and effectiveness evaluations at 4 , 8,12 , and 28 weeks; $1,1.5$, and 2 years; and annually thereafter. We report safety and effectiveness data relating to 5 years of tacrolimus maintenance therapy at the interim data cutoff in August 2016.

Patients. Patients with LN were initiated with tacrolimus (Astellas Pharma Inc.) therapy at an individualized dose, and were then maintained on tacrolimus ${ }^{16}$. The dose of tacrolimus during maintenance therapy could be adjusted for each patient on the basis of clinical signs and symptoms, aided by the monitoring of tacrolimus blood trough concentrations (determined according to local standard practice). Prednisolone (PSL) use was permitted at the discretion of the treating physician.

Study assessments. Adverse events (AE) occurring during 5-year treatment with tacrolimus were monitored. Particular attention was paid to the occurrence (based on the attending physician's assessment) of infections, renal disorders, glucose tolerance disorders, neuropsychiatric disorders, cardiac disorders, pancreatic dysfunction, malignancy (including lymphoma), worsened interstitial pneumonia, and menstrual disorders, which are identified as safety concerns of tacrolimus treatment.

Terminology from the Medical Dictionary for Regulatory Activities/ Japanese Edition (MedDRA/J; version 19.0) was used to summarize and report $\mathrm{AE}$ and adverse drug reactions (ADR) according to system organ class and preferred terms. Renal and hepatic impairment and cardiac dysfunction were defined in the study protocol (Supplementary Data 1, available with the online version of this article), and formed the basis for the physician's rating of renal, hepatic, or cardiac function as normal or abnormal. ADR were defined as AE having at least a possible relationship to the study drug, as assessed by the physician, or for which assessment of causality was missing. An ADR was considered serious if it resulted in death, was life-threatening, required hospitalization or prolongation of existing hospitalization, or resulted in persistent or significant disability/incapacity.

Effectiveness variables. Variables used to assess the effectiveness of tacrolimus maintenance therapy included cumulative rates of progression to renal failure, dialysis, and relapse (based on the attending physician's assessment according to local standard clinical practice); changes in urine protein:creatinine ratio (UPCR); serum anti-dsDNA antibody levels, serum complement $\mathrm{C} 3$ levels; and change in concomitant prednisone dose from baseline.

Statistical analyses. Categorical variables are shown as $\mathrm{n}(\%)$ and continuous variables as mean \pm SD. The tacrolimus continuation rate and cumulative incidence rates of ADR over the 5-year follow-up were calculated by survival analysis using the Kaplan-Meier method. Subgroup analyses were performed to identify factors affecting the incidence of selected ADR (including infections, renal disorders, and glucose tolerance disorders) using Fisher exact test (2 subgroups) or the Cochran-Armitage test for trend (3 or more subgroups). The log-rank and generalized Wilcoxon tests were performed to compare survival curves and used in the analysis of renal prognosis. Changes in effectiveness variables from baseline at each evaluated time point were analyzed using the Wilcoxon signed-rank test. Subgroup analyses were also performed to assess factors affecting rates of progression to renal failure, dialysis, and relapse.

All statistical comparisons were performed using 2-sided tests at the 0.05 significance level. No analyses were performed to adjust for type I error associated with multiple hypothesis testing. Missing data were not imputed in any of the analyses. In the survival assessment, patients who dropped out or were lost to follow-up without events of interest were censored at subsequent assessments. All analyses were performed using SAS (version 9.4).

Ethics. The study protocol (Supplementary Data 1, available with the online version of this article) and amendments were submitted to the Ministry of Health, Labour and Welfare (MHLW). A written agreement was obtained from participating institutions. Our study was performed in accordance with the standards for Good Post-marketing Study Practice (GPSP) provided by the MHLW in Japan. As GPSP is the authorized standard for PMS studies of approved drugs in clinical practice, no formal ethics committee approval was necessary, and informed consent was not required. To maintain privacy, all patient data were anonymized.

\section{RESULTS}

Patients. Patient disposition is presented in Supplementary Figure 1 (available with the online version of this article). Case report forms were collected for a total of 1395 patients with LN. In all, $51.5 \%$ of patients were treated at university hospitals, $20.7 \%$ at government hospitals, $14.8 \%$ at public hospitals, $4.6 \%$ at national hospitals, and $11.6 \%$ at general hospitals or clinics. Most patients were treated in rheumatology departments (67.8\%), followed by nephrology departments (28.0\%), pediatric departments (3.6\%), and dermatology departments (0.6\%).

Thirty-five patients were excluded from the safety analysis due to missing safety data, and 5 patients were not eligible for inclusion as they did not complete the survey. The remaining 1355 patients were included in this analysis based on the availability of 5-year interim data. Two patients had missing efficacy data; therefore, the effectiveness analysis set consisted of 1353 patients. The renal prognosis analysis set consisted of 1142 patients (the effectiveness analysis set, excluding 211 patients who did not receive tacrolimus continuously for at least 28 weeks, had suffered from renal failure before start of tacrolimus treatment, or for whom renal failure was diagnosed within 28 weeks after starting tacrolimus treatment).

The patients had a mean \pm SD age of $38.3 \pm 13.64$ years and were predominantly female (84.9\%). Durations of SLE and LN were $9.3 \pm 8.13$ years and $6.7 \pm 7.03$ years, respectively. Most patients had LN class IV or V (classified with the biopsy-proven pathologic type according to the International Society of Nephrology/Renal Pathology Society 2003 classification of $\mathrm{LN}^{17}$; Table 1). 
The tacrolimus continuation rate was $88.5 \%$ at Week 28 , $83.7 \%$ at 1 year and $62.7 \%$ at 5 years (Figure 1 ). Up to Week 12 , the main reason for tacrolimus discontinuation was AE. After Week 12, "unchanged/worsened symptoms" and "onset of AE" accounted for similar numbers of discontinuations. The mean \pm SD follow-up period (including additional observational period after discontinuation of tacrolimus treatment) was $1817.4 \pm$ 996.26 days in this analysis (data not shown).

Tacrolimus daily dose and blood concentration. The mean \pm SD tacrolimus daily dose was $2.22 \pm 0.825 \mathrm{mg} /$ day at baseline. The mean \pm SD daily dose was similar at Year $1(2.60 \pm 0.733 \mathrm{mg} /$ day $)$ and at Year 5 (2.58 $\pm 0.740 \mathrm{mg} /$ day). Mean tacrolimus blood concentrations were 4.3-4.7 ng/mL during Years 1-5 (Supplementary Table 1, available with the online version of this article).

Safety. Among the safety analysis population of 1355 patients, 2098 ADR were reported in 772 patients (57.0\%). The most commonly observed ADR included 83 cases (6.1\%) of hypertension, 66 cases $(4.9 \%)$ of nasopharyngitis, 59 cases $(4.4 \%)$ of upper respiratory inflammation, 55 cases $(4.1 \%)$ of diarrhea, 51 cases $(3.8 \%)$ of herpes zoster, and 45 cases $(3.3 \%)$ of bronchitis. Common serious ADR included 15 cases $(1.1 \%)$ of pneumonia, 14 cases $(1.0 \%)$ of herpes zoster, 13 cases $(1.0 \%)$ of cellulitis, and 13 cases (1.0\%) of diabetes mellitus (data not shown).

Importantly, there was no marked increase in the incidence

Table 1. Baseline demographics and clinical characteristics (safety analysis set).

\begin{tabular}{lc}
\hline & Patients, $\mathrm{N}=1355$ \\
\hline Sex & \\
Male & $205(15.1)$ \\
Female & $1150(84.9)$ \\
Age, yrs, mean \pm SD & $38.3 \pm 13.64$ \\
Duration of SLE, yrs, mean \pm SD & $9.3 \pm 8.13$ \\
Duration of LN, yrs, mean \pm SD & $6.7 \pm 7.03$ \\
Antiphospholipid syndrome & $192(14.2)$ \\
Previous treatment & \\
Mizoribine & $311(23.0)$ \\
Cyclosporine & $171(12.6)$ \\
Azathioprine & $73(5.4)$ \\
Cyclophosphamide & $72(5.3)$ \\
Mycophenolate mofetil & $30(2.2)$ \\
Pathologic type (ISN/RPS 2003 classification) & \\
Type I & $9(0.7)$ \\
Type II & $68(5.0)$ \\
Type II+V & $7(0.5)$ \\
Type III & $73(5.4)$ \\
Type III+V & $26(1.9)$ \\
Type IV-S & $64(4.7)$ \\
Type IV-S+V & $15(1.1)$ \\
Type IV-G & $149(11.0)$ \\
Type IV-G+V & $23(1.7)$ \\
Type V & $156(11.5)$ \\
Type VI & $1(0.1)$ \\
Other & $28(2.1)$ \\
\hline
\end{tabular}

Data are $\mathrm{n}$ (\%) unless indicated otherwise. G: global; ISN/RPS: International Society of Nephrology/Renal Pathology Society; LN: lupus nephritis; S: segmental; SLE: systemic lupus erythematosus. of any of the reported $\mathrm{AE}$, including serious $\mathrm{ADR}$, over the observed follow-up period (Figure 2). Of the ADR of particular interest in the safety population, infections occurred in 353 patients $(26.1 \%)$, followed by renal impairment in 137 patients (10.1\%), neuropsychiatric disorders in 87 patients (6.4\%), impaired glucose tolerance in 84 patients (6.2\%), cardiac dysfunction in 27 patients $(2.0 \%)$, malignant tumors (lymphomas) in 24 patients (1.8\%), menstrual disorders in 13 patients $(1.0 \%)$, and pancreatic dysfunction in 4 patients $(0.3 \%)$. A worsening of interstitial pneumonia was not observed.

Subgroup analysis for the incidence of ADR during the first 28 weeks of tacrolimus treatment suggested a higher risk of infection with concomitant or previous disease and in patients positive for urinary erythrocytes. A higher risk of renal disorders was seen with inpatient care, increasing age, abnormal renal or cardiac function, higher urinary protein, and higher serum creatinine. A higher risk for glucose tolerance disorders was seen with inpatient care, increasing age, higher BMI, abnormal liver function, and higher tacrolimus blood concentrations (Table 2). No significant differences were observed in any of the subgroup analyses based on LN class.

Effectiveness. In the renal prognosis analysis set, the cumulative rates of progression to renal failure were $0.8 \%$ at Year 1 and $6.6 \%$ at Year 5, while rates of progression to dialysis were $0 \%$ at Year 1 and $1.0 \%$ at Year 5. In the efficacy analysis set, the cumulative relapse rates were $7.8 \%$ at Year 1, and $30.6 \%$ at Year 5 (Figure 3).

Subgroup analysis of tacrolimus effectiveness over 5 years suggested that rates of progression to renal failure were higher in older patients and in those with higher BMI, concomitant or previous disease, abnormal renal or cardiac function, higher urinary protein, and higher serum creatinine. Rates of dialysis were higher in patients with higher BMI, abnormal renal function, and higher serum creatinine. Relapse rates were higher in inpatients, younger patients, and patients who had tested positive for urinary erythrocytes. A statistically significant association was also seen between relapse rates and mean daily steroid dose (Table 3). No significant differences were observed in any of the subgroup analyses based on LN class.

UPCR was significantly decreased from 4 weeks after the initiation of tacrolimus treatment $(P<0.001)$, and the effect was maintained at 5 years. However, no clinically or statistically significant change was observed in creatinine clearance over the follow-up period. Serum anti-dsDNA antibody and complement C3 levels were significantly improved from 4 weeks $(P<0.001)$, and the effect was also maintained to 5 years. The total daily dose of concomitant PSL fell significantly after the initiation of treatment with tacrolimus from $17.3 \pm 11.98$ to $8.5 \pm 5.25 \mathrm{mg} /$ day at 5 years $(P<0.001$; Supplementary Table 2 , available with the online version of this article).

\section{DISCUSSION}

Maintenance therapy with prolonged immunosuppressive treatment is very important in LN, owing to the high relapse rate even after successful induction treatment. Ciruelo, et al reported that successful induction treatment of $\mathrm{LN}$ with $\mathrm{CYC}$ 


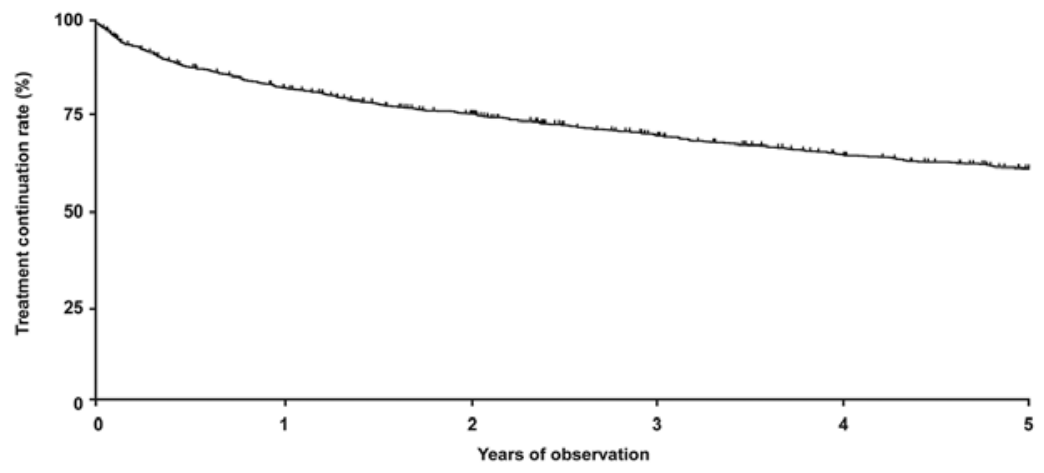

Figure 1. Treatment continuation rate with tacrolimus (efficacy analysis set).
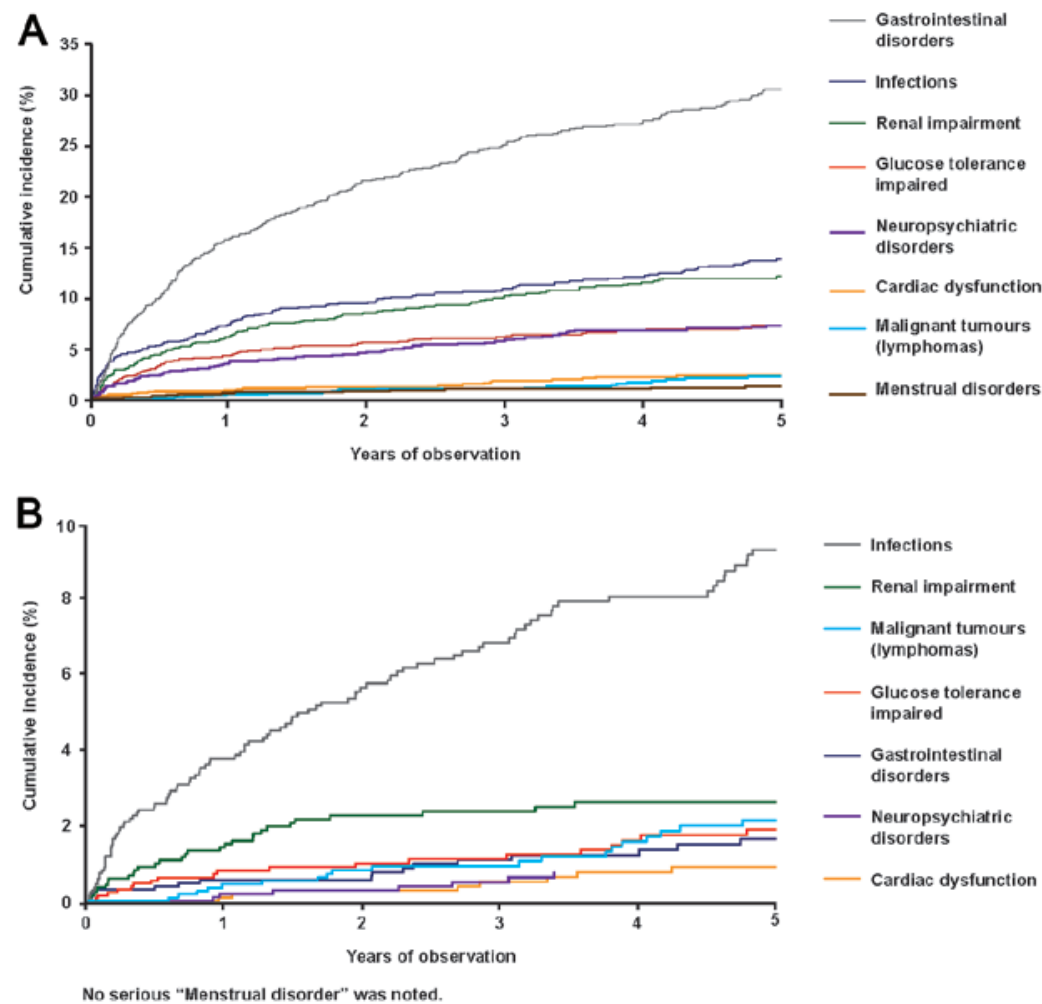

Figure 2. Cumulative incidence of adverse drug reactions, and serious adverse drug reactions (safety analysis set).

was associated with a relapse rate of approximately $25 \%$ after 5 years $^{18}$, while another report indicated that $37 \%$ of newly diagnosed patients with $\mathrm{LN}$ experienced at least 1 renal flare, despite ongoing therapy with low-dose glucocorticoids and AZA in most patients ${ }^{19}$. In the study by the Aspreva Lupus Management Study (ALMS) Group, treatment failure rates in patients with $\mathrm{LN}$ receiving maintenance therapy with MMF and AZA after successful induction therapy were $16.4 \%$ and $32.4 \%$, respectively, at 36 months ${ }^{20}$.

There is increasing evidence to suggest that the immunosuppressant tacrolimus may be an effective and well-tolerated treatment option for $\mathrm{LN}$ as both induction and maintenance therapy ${ }^{14,15,21,22,23,24,25,26}$; however, these data are largely based on short-term treatment and small patient populations. In particular, long-term data and data on its use as maintenance therapy are notably lacking. In 2014, Yap, et al reported a retrospective study of 29 patients with $\mathrm{LN}$ who received tacrolimus for 46.9 months and concluded that the effectiveness of tacrolimus warranted further investigation as a long-term maintenance agent ${ }^{27}$. 
Table 2. Subgroup analysis of the incidence of ADR occurring during 28 weeks of treatment with tacrolimus (safety analysis set).

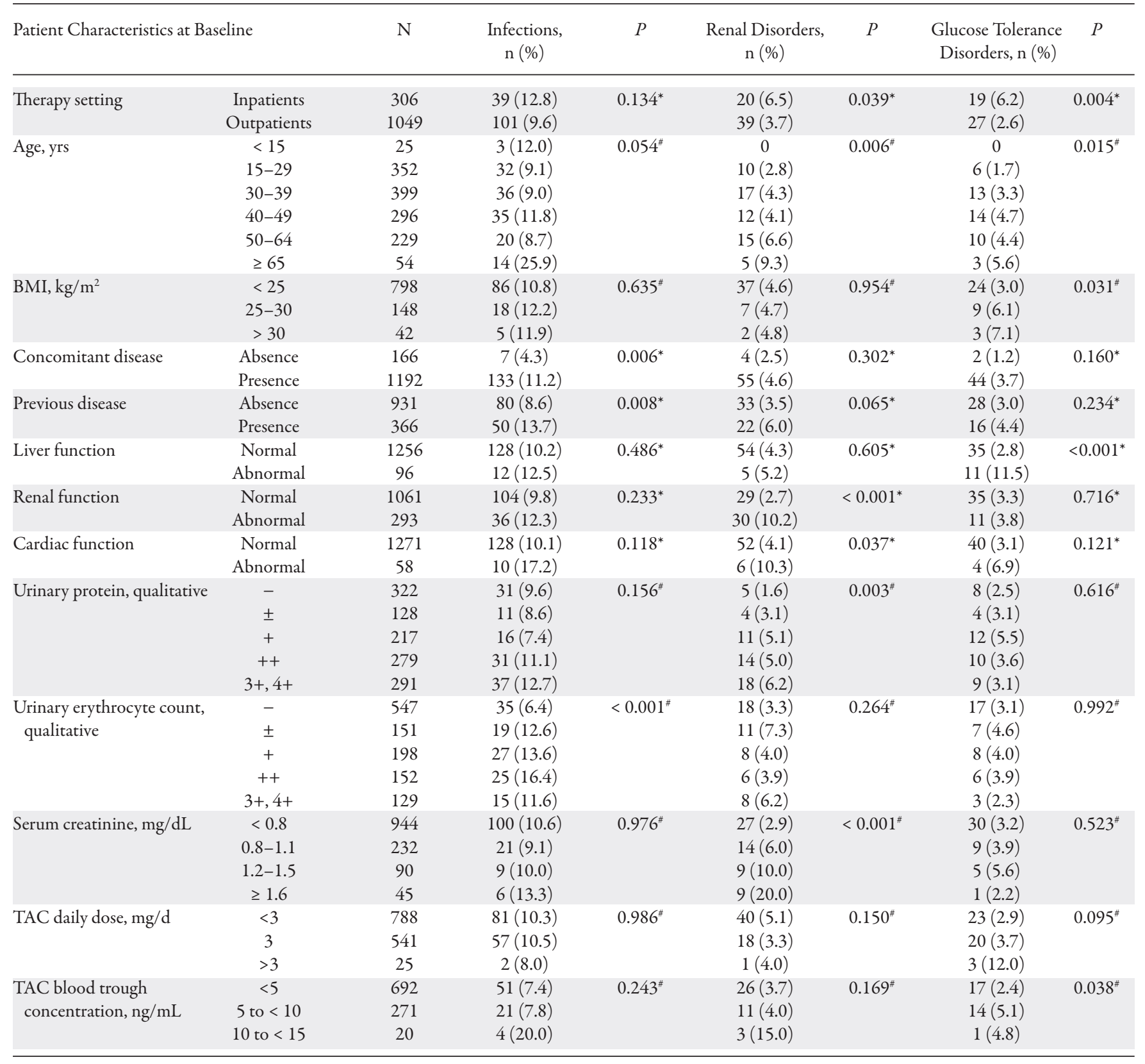

${ }^{*}$ Fisher exact test. ${ }^{*}$ Cochran-Armitage test for trend. The subgroup analyses were performed for the incidences of common ADR occurring during the first 28-weeks of treatment with tacrolimus. The incidence of infections, renal disorders, and glucose tolerance disorders were analyzed as common ADR. (-): negative; ( \pm ): pseudo-positive; (+): positive; $(++)$ : double positive; $(3+/ 4+)$ : strong positive; ADR: adverse drug reactions; TAC: tacrolimus.

In this PMS study assessing the long-term safety and effectiveness of tacrolimus as LN maintenance therapy, the tacrolimus continuation rate at 5 years was $62.7 \%$. The main reason for discontinuation of tacrolimus therapy in the early treatment period (up to Week 28) was AE, but this was a less frequent reason for discontinuation thereafter. This suggests that physicians should take care to judge whether discontinuation of tacrolimus due to $\mathrm{AE}$ is warranted, taking into consideration the duration of therapy. The tacrolimus discontinuation rate of $37.3 \%$ in the present study can be compared to that observed with other immunosuppressive agents. In a 36-month, phase III study comparing AZA and MMF as maintenance therapy for patients with LN (ALMS), the discontinuation rate due to AE was 39.6\% for AZA and 25.2\% with $M_{M}{ }^{20}$. However, lower discontinuation rates were reported at a mean follow-up of 48 months in the MAINTAIN Nephritis trial (17.3\% for AZA and $28.3 \%$ with $\mathrm{MMF})^{28}$.

The most common ADR during the 5-year tacrolimus treatment period were infections, of which nasopharyngitis, herpes zoster, and bronchitis were most frequent. The major ADR, 


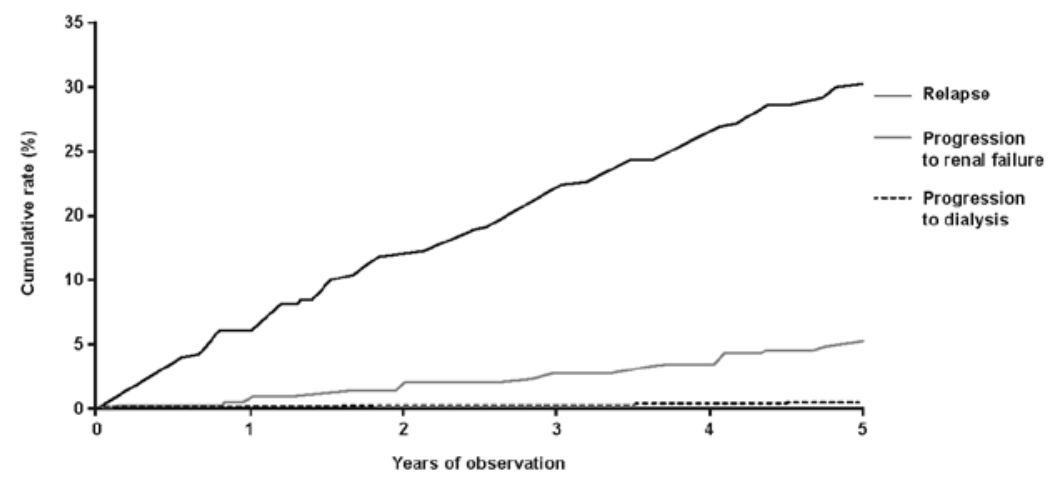

Figure 3. Cumulative rates of progression to renal failure, progression to dialysis, and relapse (renal prognosis analysis set).

including infections, tended to develop early, without any marked increase over the duration of the follow-up period. This indicates that physicians should take care to adequately explain to their patients that major ADR of tacrolimus are most likely to occur during the early treatment period and that careful monitoring for $\mathrm{ADR}$ is warranted, particularly during the initiation phase of tacrolimus treatment. There was no significant increase in the incidences of renal dysfunction and malignancy. No safety concerns with the long-term use of tacrolimus were seen in the current 5-year interim analysis.

Serum tacrolimus concentration is an important indicator for safe and effective LN management. Chen, et al reported that with titration of tacrolimus to achieve a relatively low trough blood concentration of 4-6 ng/mL, none of the tacrolimus-treated patients developed renal relapse during 6 months' maintenance therapy ${ }^{15}$. Safety profiles were favorable with very low risks of nephrotoxicity, arterial hypertension, hyperlipidemia, and other calcineurin inhibitor-related ADR ${ }^{15}$. The tacrolimus trough levels described by Chen, et al ${ }^{15}$ are likely to guide tacrolimus administration dosage and trough level monitoring in clinical practice.

Subgroup analysis of the incidence of ADR suggested that inpatient management, comorbidity or previous disease, severity of the underlying disease, increasing age, and abnormal renal or liver function were risk factors for commonly observed ADR of tacrolimus, such as infections, renal disorders, and glucose tolerance disorders. Although almost all of these are already known risk factors ${ }^{29,30}$, we believe that treatment with tacrolimus will be better tolerated if more careful attention is paid to the patients with these risk factors in real-world clinical practice.

Renal function variables were improved in the first 4 weeks after the initiation of tacrolimus therapy and were sustained to 5 years. Improvements were observed for rates of progression to renal failure, dialysis and relapse, UPCR, anti-dsDNA antibody, serum complement C3, and concomitant PSL dose. This further demonstrates stable renal function in patients with $\mathrm{LN}$ receiving long-term tacrolimus therapy in a real-world clinical practice setting, building on a previous small placebo-controlled, double-blind, multicenter study of tacrolimus therapy in LN of short duration by Miyasaka, et al ${ }^{14}$.

Our PMS study has a number of strengths. To our knowledge, it is the first prospective observational study to assess the long-term safety and effectiveness of tacrolimus for LN maintenance therapy among a large patient population in a real-world clinical setting. The study enrolled patients from 275 sites across Japan, a feature that may make it more representative of patients with LN in Asia, where substantial patient morbidity and mortality are reported ${ }^{2,3,31}$. However, we think that the data obtained from this PMS study will also be useful outside of Asia and will help to establish optimal tacrolimus treatment administration and monitoring worldwide.

Nevertheless, our study also has several limitations, including the incorrect completion of report forms, lack of protocol-defined definitions for effectiveness variables, and its open-label and noncomparative observational design, with the attendant potential for bias. Direct comparison with other standard maintenance therapies, such as AZA and MMF, is missing here and would be valuable in future studies. While our study aimed to include all patients with LN initiating maintenance treatment with tacrolimus in Japan, $14 \%$ of the target sites did not participate in the study. Without information on the number of patients receiving tacrolimus maintenance therapy for $\mathrm{LN}$ at these sites, it was challenging to estimate the overall patient coverage of the study. We believe that approximately $90 \%$ of patients with $\mathrm{LN}$ initiating maintenance treatment with tacrolimus in Japan were included. Although this is a lower proportion of patients than initially anticipated, it is considered sufficient to adequately reflect the real-world clinical setting in Japan. Finally, the results of this interim report should be regarded as preliminary in nature-as more data are collected, further analyses are planned.

In conclusion, this real-world study of tacrolimus as maintenance therapy in patients with LN showed that it is well tolerated and effective over 5 years. The final report of the ongoing PMS study at 10 years will shed further light on the clinical value and characterization of tacrolimus therapy in patients with LN. Comparative studies against other immunosuppressive therapies are also required to determine the clinical utility and role of tacrolimus in this setting.

\section{ACKNOWLEDGMENT}

The authors thank all investigators who participated in this study. Medical writing and editorial support were provided by Paola Accalai and Julia Donnelly for Cello Health MedErgy, funded by Astellas Pharma, Inc. 
Table 3. Subgroup analysis of the incidence of renal failure, rate of progression to dialysis, and relapse at Year 5 (renal prognosis analysis set).

\begin{tabular}{|c|c|c|c|c|c|c|c|c|c|}
\hline \multicolumn{2}{|l|}{$\begin{array}{l}\text { Patient Characteristics } \\
\text { at Baseline }\end{array}$} & $\begin{array}{c}\mathrm{N} \\
\\
172\end{array}$ & $\begin{array}{c}\text { Renal Failure, } \\
\text { Cumulative } \\
\text { Incidence }(95 \% \mathrm{CI}) \\
9.1(5.5-14.9) \\
6.2(4.7-8.1)\end{array}$ & $\begin{array}{c}P^{*} \\
\mathrm{I}_{1} \\
.077,0.121\end{array}$ & $\begin{array}{c}\text { Dialysis, } \\
\text { Cumulative } \\
\text { Incidence }(95 \% \mathrm{CI}) \\
0.7(0.1-4.6) \\
1.0(0.5-2.1)\end{array}$ & $\begin{array}{c}P^{*} \\
0.775,0.729\end{array}$ & $\begin{array}{c}\mathrm{N} \\
\\
204 \\
1149\end{array}$ & $\begin{array}{c}\text { Relapse, } \\
\text { Cumulative } \\
\text { Incidence }(95 \% \mathrm{CI}) \\
37.2(29.8-45.8) \\
29.4(26.4-32.7)\end{array}$ & $\begin{array}{c}P^{*} \\
0.176,0.102\end{array}$ \\
\hline Therapy setting & $\begin{array}{l}\text { Inpatients } \\
\text { Outpatients }\end{array}$ & $\begin{array}{l}236 \\
906\end{array}$ & $\begin{array}{l}4.7(2.5-8.8) \\
7.1(5.5-9.2)\end{array}$ & $0.215,0.214$ & $\begin{array}{l}1.1(0.3-4.2) \\
1.0(0.5-2.0)\end{array}$ & $0.828,0.863$ & $\begin{array}{c}306 \\
1047\end{array}$ & $\begin{array}{l}38.6(31.9-46.0) \\
28.6(25.6-32.0)\end{array}$ & $0.017,0.029$ \\
\hline $\mathrm{BMI}, \mathrm{kg} / \mathrm{m}^{2}$ & $\begin{array}{c}<25 \\
25-30 \\
30\end{array}$ & $\begin{array}{c}671 \\
119 \\
30\end{array}$ & $\begin{array}{c}5.3(3.7-7.5) \\
10.9(6.0-19.3) \\
14.6(5.7-34.3)\end{array}$ & $0.039,0.030$ & $\begin{array}{c}0.4(0.1-1.5) \\
1.1(0.2-7.8) \\
7.1(1.8-25.7)\end{array}$ & $<0.001,<0.001$ & $\begin{array}{c}797 \\
148 \\
42\end{array}$ & $\begin{array}{l}32.4(28.6-36.4) \\
29.9(22.1-39.6) \\
36.0(21.3-56.4)\end{array}$ & $0.771,0.833$ \\
\hline Previous disease & $\begin{array}{l}\text { Absence } \\
\text { Presence }\end{array}$ & $\begin{array}{l}797 \\
299\end{array}$ & $\begin{array}{c}4.2(2.9-6.0) \\
11.9(8.5-16.5)\end{array}$ & $<0.001,<0.001$ & $\begin{array}{ll}1 & 0.7(0.2-1.7) \\
& 1.6(0.6-4.1)\end{array}$ & $0.156,0.178$ & $\begin{array}{l}930 \\
366\end{array}$ & $\begin{array}{l}30.3(26.9-34.0) \\
33.9(28.5-40.1)\end{array}$ & $0.252,0.266$ \\
\hline Liver function & & $\begin{array}{c}1065 \\
74\end{array}$ & $\begin{array}{c}6.9(5.4-8.7) \\
3.7(0.9-14.0)\end{array}$ & 02680300 & $\begin{array}{c}0.9(0.5-1.9) \\
1.9(0.3-12.4)\end{array}$ & 0.5290 & $\begin{array}{c}1254 \\
96\end{array}$ & & 0.869 \\
\hline Renal function & $\begin{array}{l}\text { Normal } \\
\text { Abnormal }\end{array}$ & $\begin{array}{l}954 \\
187\end{array}$ & $\begin{array}{c}3.9(2.7-5.5) \\
20.8(15.2-28.0)\end{array}$ & $<0.001,<0.001$ & $\begin{array}{ll}1 & 0.5(0.2-1.4) \\
& 3.4(1.4-8.0)\end{array}$ & $<0.001,<0.001$ & $\begin{array}{l}1060 \\
292\end{array}$ & $\begin{array}{l}29.7(26.6-33.1) \\
34.2(27.9-41.4)\end{array}$ & $0.158,0.166$ \\
\hline Cardiac function & $\begin{array}{c}\text { Normal } \\
\text { Abnormal }\end{array}$ & $\begin{array}{c}1081 \\
39\end{array}$ & $\begin{array}{c}6.2(4.8-8.0) \\
17.9(7.8-38.1)\end{array}$ & $0.009,0.010$ & $\begin{array}{c}1.0(0.5-2.0) \\
0(0-0)\end{array}$ & $0.620,0.620$ & $\begin{array}{c}1269 \\
58\end{array}$ & $\begin{array}{l}31.2(28.2-34.3) \\
24.1(12.9-42.3)\end{array}$ & $0.766,0.603$ \\
\hline $\begin{array}{l}\text { Urinary protein, } \\
\text { qualitative }\end{array}$ & $\begin{array}{c}- \\
\pm \\
+ \\
++ \\
3+, 4+\end{array}$ & $\begin{array}{l}286 \\
116 \\
188 \\
229 \\
229\end{array}$ & $\begin{array}{c}1.8(0.7-4.7) \\
3.1(1.0-9.5) \\
7.5(4.3-12.8) \\
9.5(6.1-14.7) \\
11.6(7.8-17.0)\end{array}$ & $<0.001,<0.001$ & $\begin{array}{c}0(0-0) \\
0(0-0) \\
1.3(0.3-5.1) \\
1.7(0.6-5.2) \\
2.1(0.8-5.6)\end{array}$ & 75 & $\begin{array}{l}322 \\
128 \\
217 \\
278 \\
290\end{array}$ & $\begin{array}{l}27.6(22.3-33.9) \\
29.4(21.2-39.9) \\
26.8(20.6-34.4) \\
32.6(26.5-39.8) \\
38.8(32.2-46.1)\end{array}$ & 073 \\
\hline Steroid dose, $\mathrm{mg} / \mathrm{d}$ & $\begin{array}{c}<10 \\
10-19 \\
20-29 \\
30-39 \\
\geq 40\end{array}$ & $\begin{array}{c}555 \\
515 \\
54 \\
5 \\
3\end{array}$ & $\begin{array}{c}5.1(3.5-7.5) \\
7.8(5.6-10.8) \\
15.0(7.0-30.5) \\
0(0-0) \\
0(0-0)\end{array}$ & $0.097,0.099$ & $\begin{array}{c}0.6(0.2-2.0) \\
1.2(0.5-2.9) \\
3.0(0.4-19.6) \\
0(0-0) \\
0(0-0)\end{array}$ & $0.714,0.708$ & $\begin{array}{c}602 \\
609 \\
90 \\
19 \\
18\end{array}$ & $\begin{array}{c}19.9(16.6-23.8) \\
40.7(36.1-45.7) \\
51.8(37.7-67.6) \\
31.3(7.4-83.7) \\
20.0(3.1-79.6)\end{array}$ & $\begin{array}{l}<0.001 \\
<0.001\end{array}$ \\
\hline TAC dose, $\mathrm{mg} / \mathrm{d}$ & $\begin{array}{c}<3 \\
3 \\
>3\end{array}$ & $\begin{array}{c}765 \\
324 \\
52\end{array}$ & $\begin{array}{c}6.5(4.8-8.7) \\
7.8(5.1-11.9) \\
2.3(0.3-15.1)\end{array}$ & $0.342,0.353$ & $\begin{array}{c}0.9(0.4-2.1) \\
1.3(0.4-4.0) \\
0(0-0)\end{array}$ & $0.759,0.733$ & $\begin{array}{c}915 \\
380 \\
57\end{array}$ & $\begin{array}{l}29.1(25.7-32.7) \\
32.5(27.0-38.7) \\
43.3(30.9-58.1)\end{array}$ & $0.005,0.021$ \\
\hline
\end{tabular}

* First value: generalized Wilcoxon test; second value: log-rank test. The subgroup analyses were performed for the incidences of renal failure, rate of progression to dialysis, and relapse occurring during 5-year treatment of TAC to investigate the patient characteristics affecting the effectiveness outcomes. The Kaplan-Meier method was used to estimate cumulative incidence. $(-)$ : negative; $( \pm)$ : pseudo-positive; $(+)$ : positive; $(++)$ : double positive; $(3+/ 4+)$ : strong positive; TAC: tacrolimus.

\section{DATA AVAILABILITY}

Researchers may request access to anonymized participant level data, trial level data and protocols from Astellas sponsored clinical trials at www. clinicalstudydatarequest.com. For the Astellas criteria on data sharing see: https://clinicalstudydatarequest.com/Study-Sponsors/Study-SponsorsAstellas.aspx.

\section{ONLINE SUPPLEMENT}

Supplementary material accompanies the online version of this article.

\section{REFERENCES}

1. Mohan C, Putterman C. Genetics and pathogenesis of systemic lupus erythematosus and lupus nephritis. Nat Rev Nephrol 
2015;11:329-41.

2. Danchenko N, Satia JA, Anthony MS. Epidemiology of systemic lupus erythematosus: a comparison of worldwide disease burden. Lupus 2006;15:308-18.

3. Osio-Salido E, Manapat-Reyes H. Epidemiology of systemic lupus erythematosus in Asia. Lupus 2010;19:1365-73.

4. Korbet SM, Schwartz MM, Evans J, Lewis EJ; Collaborative Study Group. Severe lupus nephritis: racial differences in presentation and outcome. J Am Soc Nephrol 2007;18:244-54.

5. Yap DY, Lai KN. Pathogenesis of renal disease in systemic lupus erythematosus-the role of autoantibodies and lymphocytes subset abnormalities. Int J Mol Sci. 2015;16:7917-31.

6. Rahman A, Isenberg DA. Systemic lupus erythematosus. N Engl J Med 2008;358:929-39.

7. Lech M, Anders H-J. The pathogenesis of lupus nephritis. J Am Soc Nephrol 2013;24:1357-66.

8. Schwartz N, Goilav B, Putterman C. The pathogenesis, diagnosis and treatment of lupus nephritis. Curr Opin Rheumatol 2014:26:502-9.

9. Tsokos GC. Systemic lupus erythematosus. N Engl J Med 2011;365:2110-21.

10. Balow JE, Austin HA 3rd. Maintenance therapy for lupus nephritis — something old, something new. N Engl J Med 2004;350:1044-6.

11. Rovin BH, Parikh SV. Lupus nephritis: the evolving role of novel therapeutics. Am J Kidney Dis 2014;63:677-90.

12. Kuiper-Geertsma DG, Derksen RH. Newer drugs for the treatment of lupus nephritis. Drugs 2003;63:167-80.

13. Scott LJ, McKeage K, Keam SJ, Plosker GL. Tacrolimus - a further update of its use in the management of organ transplantation. Drugs 2003;63:1247-97.

14. Miyasaka N, Kawai S, Hashimoto H. Efficacy and safety of tacrolimus for lupus nephritis: a placebo-controlled double-blind multicenter study. Mod Rheumatol 2009;19:606-15.

15. Chen W, Liu Q, Chen W, Tang X, Fu P, Liu F, et al. Outcomes of maintenance therapy with tacrolimus versus azathioprine for active lupus nephritis: a multicenter randomized clinical trial. Lupus 2012;21:944-52.

16. Prograf [Package insert for Capsules $0.5 \mathrm{mg}$ and $1 \mathrm{mg}$ (tacrolimus hydrate)]. Astellas Pharma Inc.

17. Weening JJ, D’Agati VD, Schwartz MM, Seshan SV, Alpers CE, Appel GB, et al. The classification of glomerulonephritis in systemic lupus erythematosus revisited. Kidney Int 2004;65:521-30.

18. Ciruelo E, de la Cruz J, López I, Gómez-Reino JJ. Cumulative rate of relapse of lupus nephritis after successful treatment with cyclophosphamide. Arthritis Rheum 1996;39:2028-34.
19. El Hachmi M, Jadoul M, Lefebvre C, Depresseux G, Houssiau FA. Relapses of lupus nephritis: incidence, risk factors, serology and impact on outcome. Lupus 2003;12:692-6.

20. Dooley MA, Jayne D, Ginzler EM, Isenberg D, Olsen NJ, Wofsy D, et al. Mycophenolate versus azathioprine as maintenance therapy for lupus nephritis. N Engl J Med 2011;365:1886-95.

21. Tanaka H, Oki E, Tsuruga K, Yashiro T, Hanada I, Ito E. Management of young patients with lupus nephritis using tacrolimus administered as a single daily dose. Clin Nephrol 2009;72:430-7.

22. Chen W, Tang X, Liu Q, Chen W, Fu P, Liu F, et al. Short-term outcomes of induction therapy with tacrolimus versus cyclophosphamide for active lupus nephritis: a multicenter randomized clinical trial. Am J Kidney Dis 2011;57:235-44.

23. Li X, Ren H, Zhang Q, Zhang W, Wu X, Xu Y, et al. Mycophenolate mofetil or tacrolimus compared with intravenous cyclophosphamide in the induction treatment for active lupus nephritis. Nephrol Dial Transplant 2012;27:1467-72.

24. Mok CC, Tong KH, To CH, Siu YP, Au TC. Tacrolimus for induction therapy of diffuse proliferative lupus nephritis: an open-labeled pilot study. Kidney Int 2005;68:813-7.

25. Bao H, Liu ZH, Xie HL, Hu WX, Zhang HT, Li LS. Successful treatment of class V+IV lupus nephritis with multitarget therapy. J Am Soc Nephrol 2008;19:2001-10.

26. Mok CC, Ying KY, Yim CW, Siu YP, Tong KH, To CH, et al. Tacrolimus versus mycophenolate mofetil for induction therapy of lupus nephritis: a randomised controlled trial and long-term follow-up. Ann Rheum Dis 2016;75:30-36.

27. Yap DY, Ma MK, Mok MM, Kwan LP, Chan GC, Chan TM. Long-term data on tacrolimus treatment in lupus nephritis. Rheumatology 2014;53:2232-37.

28. Houssiau FA, D’Cruz D, Sangle S, Remy P, Vasconcelos C, Petrovic R, et al. Azathioprine versus mycophenolate mofetil for long-term immunosuppression in lupus nephritis: results from the MAINTAIN Nephritis Trial. Ann Rheum Dis 2010;69:2083-89.

29. Takahashi K, Abe R, Usuki S, So M. Safety and efficacy of once-daily modified-release tacrolimus in kidney transplant recipients: interim analysis of multicenter postmarketing surveillance in Japan. Transplant Proc 2014;46:406-10.

30. Takeuchi T, Kawai S, Yamamoto K, Harigai M, Ishida K, Miyasaka N. Post-marketing surveillance of the safety and effectiveness of tacrolimus in 3,267 Japanese patients with rheumatoid arthritis. Mod Rheumatol 2014;24:8-16.

31. Yap DYH, Chan TM. Lupus nephritis in Asia: clinical features and management. Kidney Dis 2015;1:100-9. 